\title{
Eficiencia del tratamiento de casos nuevos de tuberculosis sensible en Chile
}

\author{
CARLOS PEÑA M.* y NADIA ESCOBAR S.**
}

\section{Treatment efficiency in new cases of susceptible tuberculosis in Chile}

Tuberculosis therapy with the primary regimen recommended by the World Health Organization does not cure all cases globally, but it reached success in at least $85 \%$ of cases in the year 2018. The same year in Chile, treatment efficiency is achieved in only $76 \%$, mainly due to the high proportion of deaths and loss of follow-up during therapy. Preliminary data show that in the 2019 cohort the success was achieved only in about $74 \%$ of new cases. Treatment failures in Chile are rare due to national surveillance of drug susceptibility. To reduce fatality, it is necessary to reinforce the strategies for early diagnosis of tuberculosis through new algorithms. Such strategies should include molecular biology and radiology in suspected TB cases, to promote proper management of comorbidities, establish an adequate social support network and have centers available for prolonged hospitalization when needed. In addition, patient's adherence to therapy should be strengthened with strategies that encourage and facilitate attendance.

Key words: Tuberculosis; Follow Up Studies; Cohort studies; Treatment Failure.

\section{Resumen}

La terapia de la tuberculosis con el esquema primario recomendado por la OMS no logra la curación de todos los casos a nivel mundial, pero en general alcanza un éxito de curación de al menos el 85\% de los casos en el año 2018. El mismo año en Chile la eficiencia del tratamiento es solo de $76 \%$, principalmente por la alta proporción de muertes y pérdida de seguimiento durante la terapia. Datos preliminares muestran que la cohorte ingresada en 2019 tuvo un éxito de tratamiento cercano a 74\%. En Chile los fracasos de tratamiento son infrecuentes, debido principalmente a la vigilancia nacional de la susceptibilidad a fármacos. Para reducir la letalidad es necesario reforzar las estrategias para el diagnóstico precoz de la tuberculosis, mediante nuevos algoritmos que incorporen la biología molecular y la radiología en casos sospechosos de esta enfermedad, fomentar el adecuado manejo de las comorbilidades, establecer una adecuada red de apoyo social y disponer de centros de hospitalización cuando se requieren. Además, se debe fortalecer la adherencia a la terapia de los pacientes con estrategias de incentivo y facilitación de la asistencia.

Palabras clave: Tuberculosis; estudios de seguimiento; estudios de cohorte; fracaso del tratamiento.

\section{Eficiencia de la terapia primaria de la tuberculosis en el mundo}

El control de la tuberculosis (TBC) se logra identificando los casos pulmonares que trasmiten la enfermedad y administrando una terapia, reco- mendada por la Organización Mundial de la Salud (OMS) en base a una sólida evidencia científica de la eficacia, para los casos nuevos sensibles al esquema propuesto, con la cual se deberían curar todos los pacientes. Este esquema, llamado "primario", utiliza 4 fármacos: Isoniazida $(\mathrm{H})$,

* Encargado Programa de Control y eliminación de la Tuberculosis (PROCET) del Servicio de Salud Metropolitano Central. Hospital Clínico San Borja Arriarán.

** Directora Nacional del PROCET. Ministerio de Salud de Chile. 
Rifampicina (R), Etambutol (E) y Pirazinamida (Z) administrados diariamente hasta completar 50 dosis y posteriormente $\mathrm{H}$ y $\mathrm{R}$ diario (en Chile trisemanal) hasta completar 48 dosis $^{1}$. La OMS propone alcanzar como meta una curación de al menos el 90\% de los afectados. Espera, además, que la proporción de pacientes que abandonan la terapia (pérdida de seguimiento) sea inferior al 5\% y los fallecidos no sobrepasen el 3\%. La OMS registra en un reporte anual los resultados de las cohortes de tratamiento agrupadas en distintas regiones del mundo. Estas cohortes consideran casos de TBC nuevos y recaídas en conjunto, casos con VIH asociado y casos con resistencias. Al observar estos informes entre los años 2012 y 2018 se puede concluir que el promedio de la curación para el período de estos 7 años es de $84 \%$, con la mayor curación $(86 \%)$ los años 2012 y 2013 y la menor curación (81\%) el año $2016^{2}$ (Figura 1).
En pacientes con VIH, el promedio de curación en dicho período es mucho menor, alcanzando el 74\%. La mayor proporción de curación (78\%) se observa el año 2015 y la menor curación el año 2012 (68\%) (Figura 2).

El reciente informe de resultados del tratamiento de la TBC a nivel mundial realizado por la OMS consigna que, durante el año 2018, la curación promedio mundial fue de $85 \%{ }^{2}$. En este informe se establece que la región con el mejor promedio de curación ese año alcanza el 91\% (región del Mediterráneo Este). Sin embargo, existen regiones con alta proporción de fracasos (región Europea con 11\%), otras con alta proporción de muertes (región del Sudeste Asiático con $17 \%$ ) y otras con alta proporción de 'pérdida de seguimiento' (región del Pacífico Oeste con $26 \%$ ), reflejando diferentes motivos por los cuales no se alcanza la meta propuesta de curación. Los pacientes con VIH logran en promedio una
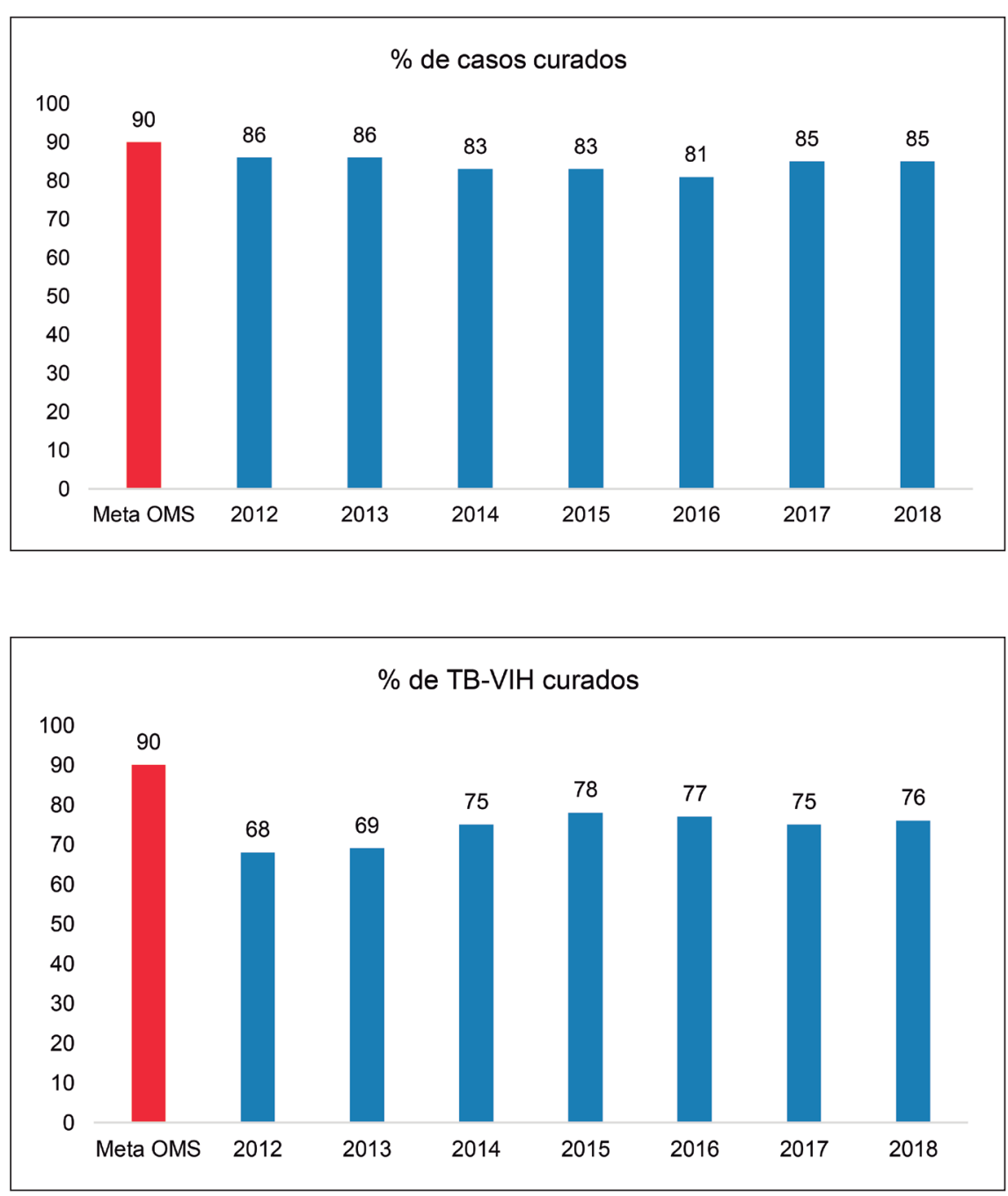

Figura 1. Porcentaje de curación de TBC (casos nuevos y recaídas) en el mundo (2012-2018). Se compara las cifras con la meta de la OMS (primera columna).
Figura 2. Porcentaje de curación de TBC-VIH mundial (casos nuevos y recaídas) (2012-2018). Se compara las cifras con la meta de la OMS (primera columna). 
curación de $76 \%$ y una letalidad de $11 \%$. La región de Las Américas en este informe alcanza una curación de $76 \%$ en los casos nuevos y recaídas, mientras que la curación es de sólo $56 \%$ para los casos con VIH.

\section{La curación de la tuberculosis con esquema primario en Chile}

El esquema recomendado por la OMS se ha adoptado en nuestro país desde hace más de 2 décadas para administrarlo en los casos nuevos sensibles a los fármacos que contiene el esquema. Este tratamiento se entrega en forma gratuita a los pacientes mediante una administración controlada en forma directa por un miembro del equipo del Programa de Control y Eliminación de la TBC (PROCET) capacitado. Esta estrategia se conoce como DOT (directly observed treatment). En Chile, la meta de curación definida por el PROCET es $90 \%{ }^{3}$.

Las cohortes evaluadas en nuestro país consideraban los casos nuevos de adultos (mayores de 15 años) con TBC pulmonar bacilífera (diagnóstico mediante baciloscopía) hasta el año 2013. Posteriormente, en la evaluación de las cohortes de tratamiento se incluyeron todos los casos de TBC independiente de su localización pulmonar o extrapulmonar, sin resistencias a fármacos. Se dispone de datos correspondientes a los años 2008 a $2019^{4}$.

En el período comprendido entre los años 2008 y 2013, en la cohorte de casos pulmonares bacilíferos, el promedio de curación es de 80,6\%. Mientras que en el período entre el año 2014 y
2018, el promedio de curación es de 75,9\%, en los casos de todas las formas de tuberculosis. Debemos señalar que para el año 2014 no se dispone de la información del $21,2 \%$ de los casos y la curación por esta razón queda reducida a $60,7 \%$. Para efectos de evitar un valor no fidedigno se distribuyeron los casos no informados de acuerdo con la proporción de las cifras basales conocidas de esa cohorte en las diferentes categorías. De esta manera, la curación para ese año se corrige a $73,4 \%$, lo que resulta más consistente con los valores establecidos en las cohortes previas.

La última evaluación de la cohorte del año 2019 de casos nuevos sensibles al esquema primario informada provisoriamente por el PROCET, señala una curación de $74,2 \%$ (existe $7,6 \%$ de casos no informados por lo que la curación en el mejor de los casos no superaría el 81,8\%). La eficiencia de la curación en el país entre los años 2008 y 2019 (considerando que el período 20082010 es un promedio del trienio) muestras cifras inferiores a la meta nacional (Figura 3).

Los pacientes no completan su terapia porque una proporción fallece antes de terminar su terapia o abandonan la terapia sin concluirla (pérdida de seguimiento), mientras que una muy pequeña proporción presenta fracaso del tratamiento $(<1 \%)$. En la última evaluación nacional de la cohorte del año 2019 la proporción de fallecidos es de 9,3\% y las pérdidas de seguimiento de $7,3 \% \%^{5}$. La proporción de abandonos entre los años 2008 y 2019 no cumple con la meta esperada (Figura 4). Por último, la proporción de muertes durante el tratamiento en esos períodos señalados tampoco cumple con la meta nacional propuesta (Figura 5).

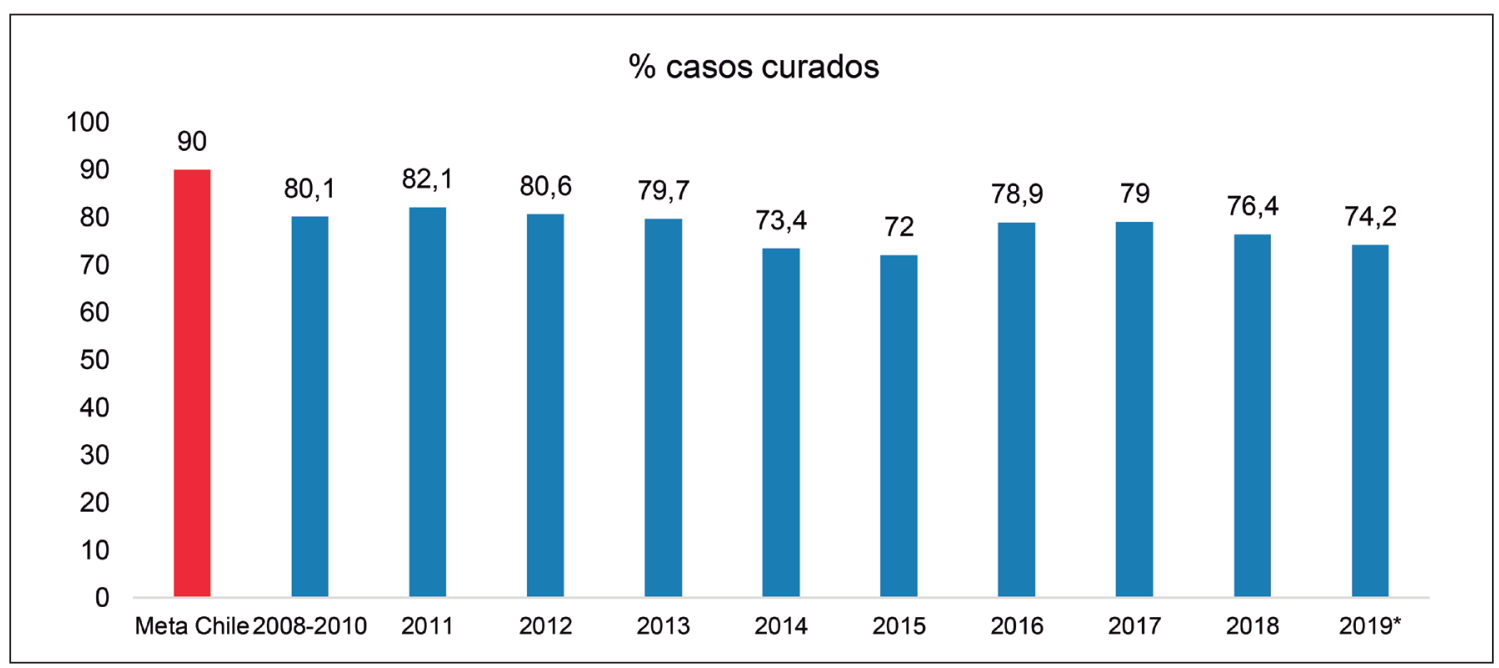

Figura 3. Porcentaje de curación de casos nuevos de TBC en Chile. (2008-2019). *Datos 2019 son preliminares. Se compara las cifras con la meta nacional (primera columna). 

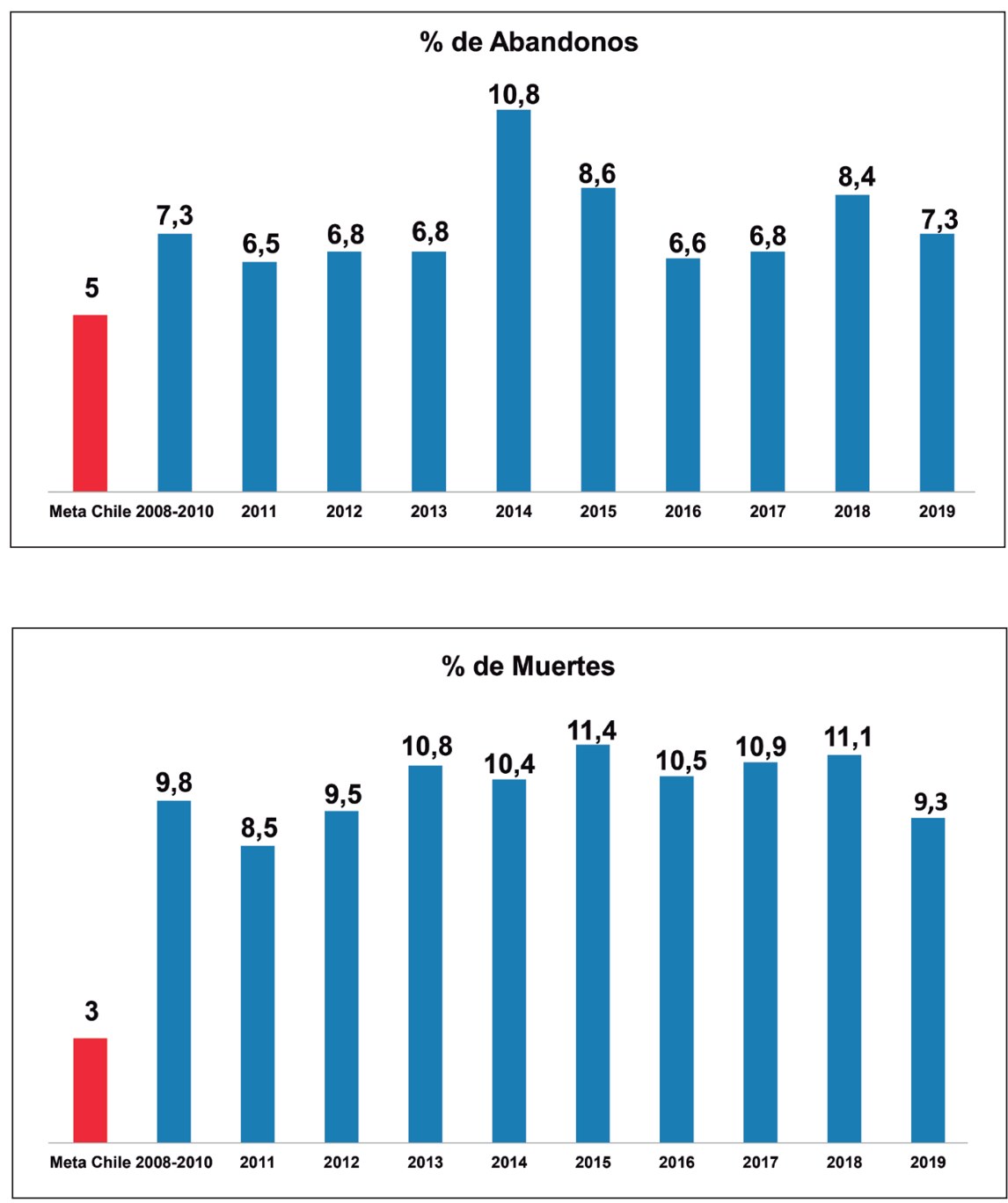

Figura 4. Porcentaje de abandono de tratamiento de casos nuevos de TBC en Chile. (2008-2019). Datos 2019 son preliminares. Se compara las cifras con la meta nacional (primera columna).
Figura 5. Porcentaje de muertes de casos nuevos de TBC en Chile. (20082019). Datos 2019 son preliminares. Se compara las cifras con la meta nacional (primera columna).

\section{Factores relacionados con la curación de la tuberculosis}

\section{Inmunodepresión por VIH}

Como ya se ha informado en esta revisión, los pacientes con VIH alcanzan una menor proporción de curación ( $76 \%$ a nivel mundial el año 2018). En la región de las Américas la curación sólo llegaría al 56\%. En nuestro país se puede acceder a los resultados de la curación en pacientes con VIH a partir de las cohortes del año 2014 en adelante y se encuentra reportado en los informes anuales del programa nacional de TBC a partir del año $2015^{4}$. La cohorte del quinquenio 20142018 registra un promedio de curación de 52,8\%, con la menor curación $(45,6 \%$ en la cohorte del año 2014) y la mayor curación $(63,3 \%)$ en los pacientes del año 2017 (Figura 6).

En ese mismo quinquenio, el promedio de la proporción de fallecidos alcanza a 20,9\% con la mayor letalidad el año $2015(26,7 \%)$ y la menor letalidad el año 2014 (14,7\%) (Figura 7).

La pérdida de seguimiento alcanza un promedio de $17,1 \%$ en ese quinquenio, con la mayor proporción de estos abandonos el año 2018 $(19,3 \%)$ y la menor proporción de abandonos el año 2014 (15,7\%) (Figura 8).

La última información (provisoria) para la cohorte del año 2019 indica una curación de 59,5\%, con una alta proporción de fallecidos durante la terapia $(21,6 \%)$ y una proporción algo menor, pero aún elevada, de pérdida de seguimiento de los pacientes $(10,6 \%)$. Los fracasos son muy ocasionales $(1,3 \%)^{5}$.

En pacientes con TBC y VIH la letalidad es uno de los principales factores que reducen la curación. Con frecuencia su cuadro clínico es avanzado y las formas clínicas diseminadas y a 
Figura 6. Porcentaje de curación de casos nuevos de TB-VIH en Chile. (2014-2019). Datos 2019 son preliminares. Se compara las cifras con la meta nacional (primera columna).

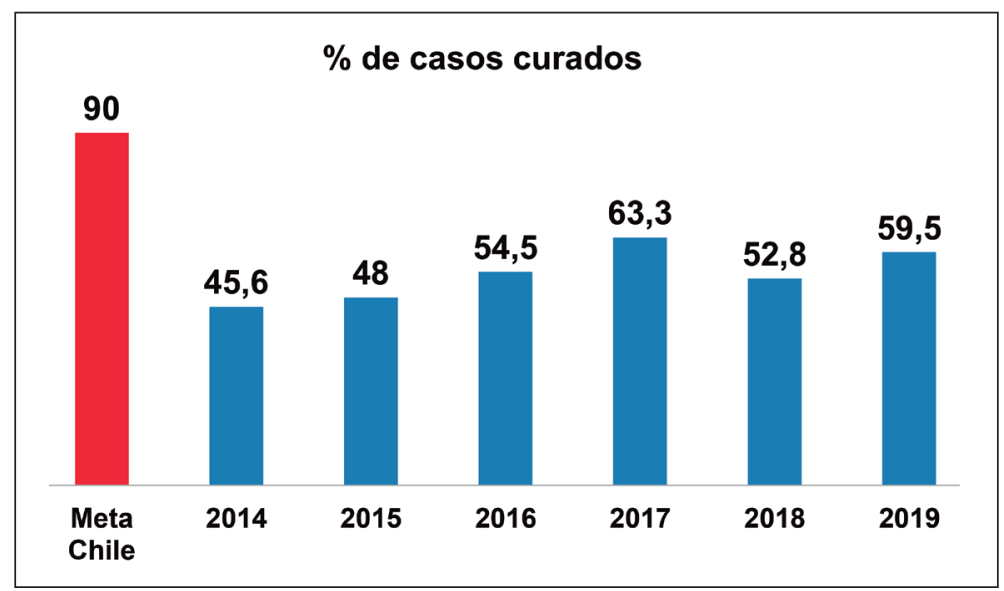

Figura 7. Porcentaje de muertes de casos nuevos de TB-VIH en Chile. (2014-2019). Datos 2019 son preliminares. Se compara las cifras con la meta nacional.

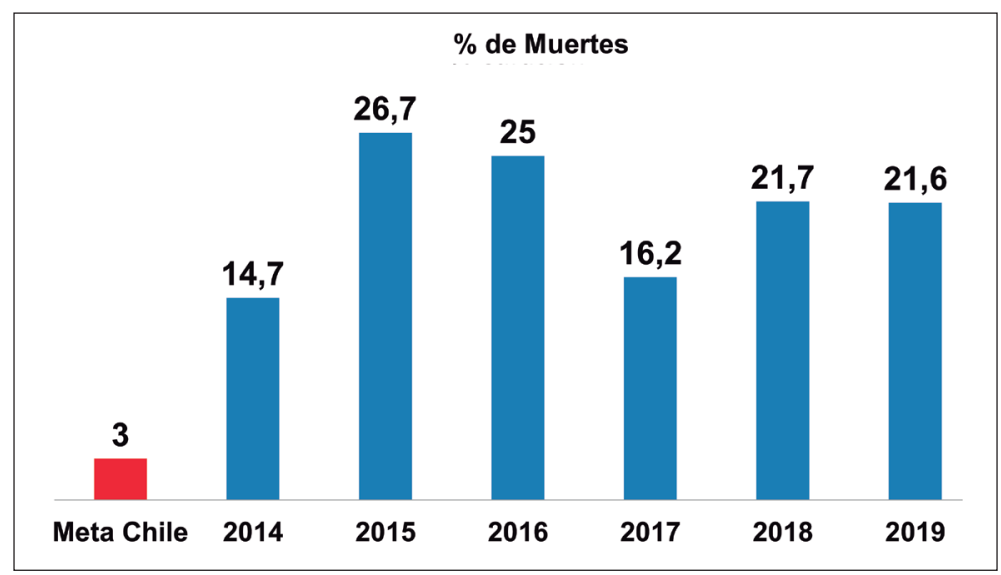

Figura 8. Porcentaje de abandono de tratamiento de casos nuevos de TBVIH en Chile. (2014-2019). Datos 2019 son preliminares. Se compara las cifras con la meta nacional (primera columna).

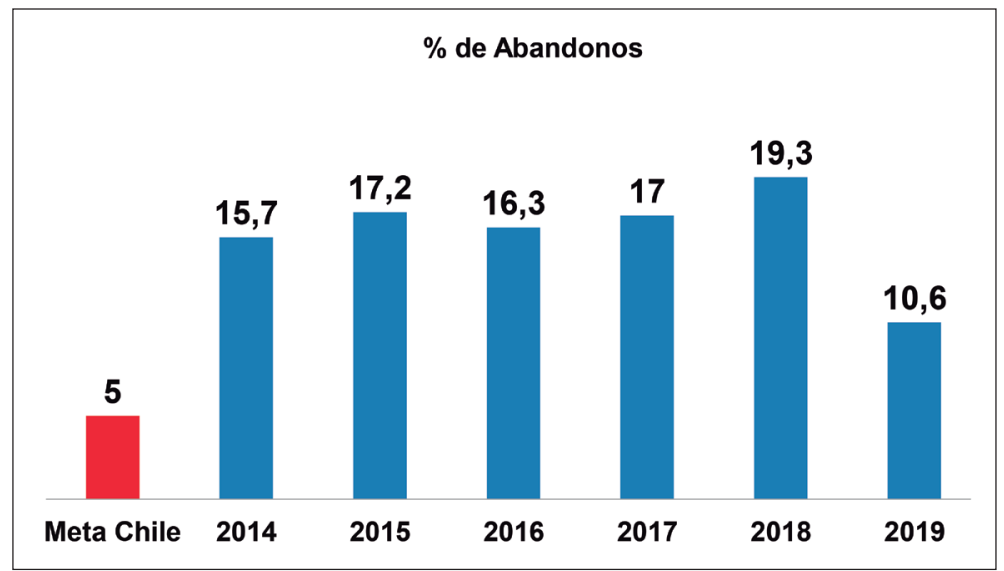

menudo se asocian a otras infecciones oportunistas y comorbilidades, además es habitual el uso de múltiples fármacos con potencial de interacciones y efectos colaterales. Hay que destacar la ausencia de fracasos en estos pacientes lo que hace pensar que la resistencia a los fármacos no es un factor relevante.

\section{Resistencia a fármacos}

Otros aspectos interesantes de comentar, que podrían reducir la eficacia en la curación son la presencia de casos resistentes a $\mathrm{H}$, que no son diagnosticados inicialmente y que pueden ocasionar multidrogoresistencia al exponer a monoterapia con R a estos pacientes; sin embargo, los pro- 
tocolos de utilización de biología molecular (line probe assay) para la determinación de genes de resistencia a H (InhA, KatG) a partir del cultivo o de la muestra inicial instalado en Chile, ha logrado identificar a estos pacientes. La resistencia a fármacos no sería un factor incidente en la baja curación de nuestros pacientes, ya que los fracasos se presentan en menos del 1\%. La mayoría de nuestros casos con monorresistencia a $\mathrm{H}, \mathrm{R}$ o MDR son casos de resistencia inicial, de acuerdo a la vigilancia universal de la susceptibilidad que efectúa el Instituto de Salud Pública de Chile.

\section{Biodisponibilidad de fármacos}

Se ha discutido bastante los aspectos relacionados con la biodisponibilidad de los fármacos. $\mathrm{El}$ caso de la $\mathrm{R}$ es trascendente ya que es el fármaco esencial del esquema primario. Su dosis habitual es de $10 \mathrm{mg} / \mathrm{kg}$ con un máximo de $600 \mathrm{mg}$ diarios y su 'concentración inhibitoria mínima' (CIM) es de 8,2-24 $\mu \mathrm{g} / \mathrm{ml}^{6,7}$. Con la dosis descrita se ha encontrado en algunos casos (pacientes con $\mathrm{VIH}$, infección por criptosporydium, diabéticos y mala absorción e incluso sujetos sanos) niveles plasmáticos de $\mathrm{R}$ bajo el nivel recomendado para una adecuada $\mathrm{CIM}^{8,9,10}$. Esto ha promovido líneas de investigación de terapias con mayores dosis de $\mathrm{R}$ incluso hasta $35 \mathrm{mg} / \mathrm{kg}$ con buena tolerancia y sin mayor proporción significativa de efectos colaterales. Estas investigaciones han demostrado una reducción en el tiempo de negativización del cultivo, menor tasa de recaídas, menor mortalidad y por supuesto mayor proporción de curación ${ }^{11,12,13,14,15,16}$. Sin embargo, esperamos más estudios farmacológicos respecto de la biodisponibilidad de la $\mathrm{R}$ en los diferentes tejidos, junto con investigaciones que permitan conocer el aporte $\mathrm{y}$ papel en la protección de la resistencia con el uso de altas dosis, así como disponer de estudios de seguimiento en el tiempo de los pacientes para conocer la tasa de recaídas con estas mayores dosis. Se están desarrollando investigaciones en terapia fármaco-genómica guiada por niveles plasmáticos y modelos fármaco-cinéticos ${ }^{17,18,19,20}$.

Para el clínico es importante considerar, calificar y monitorear en forma individual a todos los pacientes bajo terapia, ya que algunos podrán presentar grandes lesiones cavitarias con alta carga de población bacilar, otros pacientes pueden tener una respuesta bacteriológica de conversión de los cultivos más lenta sin albergar cepas resistentes, otros pueden estar afectados por formas de TBC extra-pulmonares que requieren mayores tiempos de terapia (osteo-articular, meníngea), otros pueden también estar afectados por inmunodepresión severa (pacientes con VIH y CD4 muy bajos, Quimioterapias, terapias biológicas, etc.) y podrían requerir terapias con mayor exposición a los fármacos (terapia diaria completa incluso con mayores dosis), estas particularidades se deben resolver en los comités de especialistas.

\section{Letalidad de la TBC}

Finalmente, para lograr una meta adecuada de curación es necesario intervenir los aspectos relacionados con la alta letalidad, donde probablemente el diagnóstico tardío, las comorbilidades y las deficiencias en las redes de apoyo de los pacientes son los factores más gravitantes. Se ha descrito previamente la alta proporción de muertes durante el tratamiento de la TBC. Las auditorias locales revelan que en casi la mitad de estas muertes la TBC no era la causa directa de este evento. La enfermedad avanzada asociada a la deficitaria situación socioeconómica (situación de calle, pobreza), trastornos mentales y adicción a drogas y alcohol, además de la presencia de otras comórbilidades $(\mathrm{VIH}$, enfermedades crónicas en adultos mayores como diabetes, enfermedad renal crónica, daño hepático crónico) en pacientes con baja o irregular asistencia a la terapia, suelen vincularse con un desenlace fatal. Con frecuencia, los casos que fallecen corresponden a formas graves y avanzadas de TBC. Actualmente, se promueve una búsqueda "activa" de casos de TBC más que una pesquisa pasiva, por lo que se ha incorporado al paciente sintomático respiratorio consultante otro perfil de búsqueda, que es el "sospechoso" de tuberculosis (paciente con fiebre prolongada, baja de peso, persistencia de tos, imágenes radiológicas patológicas, etc.). En el abanico de la búsqueda de TBC ahora disponemos de la biología molecular, que entrega una sensibilidad mejor que la baciloscopía y similar al cultivo de Koch, con la diferencia de sus rápidos resultados y de la entrega de susceptibilidad a fármacos, habitualmente a la R. Junto a esto, existe una mayor utilización de recursos imagenológicos, procedimientos (lavado broncoalveolar, biopsias, punciones, etc.) que pueden combinarse con la biología molecular ${ }^{21,22,23,24,25,26,27,28,29}$.

\section{Abandono de la terapia de la TBC}

Los altos niveles de abandono se encuentran relacionados con la educación, motivación y compromiso tanto de los pacientes como del equipo tratante y también de los factores socioeconómicos. Las dificultades para asistir a los centros de terapia ya sea por el costo de transporte, distancia y tiempo necesarios de desplazamiento, interferencias laborales y estigmatización del paciente, van originando una fuerza suficiente 
para provocar el abandono del tratamiento, cuando el paciente se percibe en mejores condiciones de salud, o cuando presenta algún efecto colateral indeseado o una reacción adversa a los fármacos. En los pacientes extranjeros, se agrega una mayor movilidad de sus residencias y lugares de trabajo, con viajes que obligan a trasladar a los pacientes a otros centros de terapia, cuando por supuesto esta movilidad es anticipada y conocida por el equipo de salud. De otra manera, el paciente es difícil de localizar para continuar su terapia.

\section{Comentarios finales}

Disponemos de fármacos muy eficaces para la terapia de la TBC. Sin embargo, este sólo hecho no garantiza la curación. Los factores que limitan la curación pueden resultar acaso algo simples de identificar, pero son difíciles de intervenir con la sola acción del sector salud. Es necesario el trabajo en equipo multidisciplinario, el manejo integral del paciente, el abordaje social y económico, la participación de facilitadores inter-culturales, agentes comunitarios, colectivos de voluntarios y el involucramiento de la comunidad. Antes de pensar en cambios o modificaciones de fármacos, es necesario considerar que lo más relevante para alcanzar una adecuada proporción de curación de nuestros pacientes es reducir la mortalidad y asegurar la asistencia, monitoreo y vigilancia del cumplimiento del curso de la terapia. Recomendamos las siguientes estrategias para reducir la letalidad: hospitalizar a los casos que lo requieren, manejar las comorbilidades como ajustar las dosis de hipoglicemiantes, corticoides, anticoagulantes, anticonvulsivantes y una innumerable cantidad de otros fármacos que podrían ver afectado su nivel plasmático o su efecto debido al uso de $\mathrm{R}$, evitar las interacciones indeseadas ( $\mathrm{R}$ con inhibidores de la proteasa en pacientes con VIH, H con antimicóticos que potencia una mayor probabilidad de daño hepático, etc.), mejorar el estado nutricional y psicológico del paciente y mantener capacitado al personal sanitario a cargo de estos pacientes. Esto, por supuesto, acompañado de una estrategia de diagnóstico precoz. Para evitar el abandono o asistencia irregular del paciente, sugerimos evaluar inicialmente la probabilidad de este evento indagando por los factores involucrados al abandono e intervenirlos, como es el caso de los problemas mentales y adicciones, en los cuales los pacientes deben ser derivados a los programas de Salud Mental, los problemas laborales y sociales mediante la trabajadora social, el apoyo de intérpretes o traductores, de asociaciones y de colectividades para extranjeros y pueblos originarios. Además debemos considerar otros apoyos prácticos, como proveer ayuda al transporte a centros de terapia (vehículo municipal, subsidios y ayudas, etc.) especialmente en la ruralidad, el entrenamiento de otros profesionales idóneos para la administración domiciliaria de los fármacos, el apoyo de hospitalización domiciliaria y programa de postrados, la salida del equipo técnico a terreno, etc. Los factores asociados a una buena relación entre las personas a menudo resultan ser esenciales. El entorno de afecto, paciencia, atención y dedicación que otorga el equipo sanitario promueve una mayor adherencia del paciente indudablemente. Si aplicamos estas estrategias tendremos mayor posibilidad de éxito en los tratamientos de la Tuberculosis.

\section{Bibliografía}

1.- WORLD HEALTH ORGANIZATION. Guidelines for treatment of drug-susceptible tuberculosis and patient care, 2017 update. Geneva; 2017.

2.- WORLD HEALTH ORGANIZATION. Global TB report 2020.

3.- MINISTERIO DE SALUD DE CHILE. Normas Técnicas para el Control y la Eliminación de la Tuberculosis. 2014. Subsecretaría de Salud Pública. División de Prevención y Control de Enfermedades. Programa Nacional para el Control y la Eliminación de la Tuberculosis.

4.- MINISTERIO DE SALUD DE CHILE. Informe anual de situación epidemiológica y operacional del programa Nacional de Tuberculosis años 2011 al 2019. www. minsal.cl

5.- MINISTERIO DE SALUD DE CHILE. Informe provisorio en seminario nacional de Tuberculosis. 24 de marzo, 2021. Programa Nacional de Control y Eliminación de la Tuberculosis.

6.- MOUTON RP, MATTIE H, SWART K, KREUKNIET J, DE WAEL J. Blood levels of rifampicin, desacetylrifampicin and isoniazid during combined therapy. J. Antimicrob Chemother. 1979; 5: 447-54.

7.- MITCHISON DA. Role of individual drugs in the chemotherapy of tuberculosis. Int. J. Tuberc. Lung Dis. 2000; 4: 796-806.

8.- SAHAI JK, GALLICANO L, SWICK S, TAILOR G, GARBER I, SEGUIN, et al. Reduced plasma concentrations of antituberculosis drugs in patients with HIV-infection. Ann. Intern. Med.1997; 127: 289-93.

9.- KIMERLING M, PHILliPS P, PATTERSON P, HALL M, ROBINSON A, DUNLAP N. Low serum antimycobacterial drug levels in non-HIV-infected tuberculosis patients. Chest 1998; 113: 1178-83.

10.- MCILLERON H, WASH P, BURGER A, NORMAN 
J, FOLB PI, SMITH P. Determinants of rifampin, isoniazid, pyrazinamide, and ethambutol pharmacokinetics in a cohort of tuberculosis patients. Antimicrob Agents Chemother. 2006; 50 (4): 1170-7. doi: 10.1128/ AAC.50.4.1170-1177.2006.

11.- STEINGART KR, JOTBLAD S, ROBSKY K, DECK D, HOPEWELL PC, HUANG D, et al. Higher-dose rifampin for the treatment of pulmonary tuberculosis: a systematic review. Int J Tuberc Lung Dis. 2011; 15 (3): 305-16.

12.- SVENSSON RJ, NIWARD K, FORSMAN LD, BRUCHFELD J, PAUES J, ELIASSON E, et al. Individualised dosing algorithm and personalised treatment of high-dose rifampicin for tuberculosis. Br J Clin Pharmacol. 2019; 85 (10): 2341-50. doi: 10.1111/ bcp. 14048 .

13.- HUY LIU A, ORTEGA-MURO F, ALAMEDAMARTIN L, MITCHISON D, COATES A. High dose Rifampicin Kills persisters, shorten treatment duration and reduces relapses rate in vitro and in vivo. Front Microbiol; 6: 641 doi: 10.3389/fmich.2015.00641

14.- BOEREE MJ, DIACON AH, DAWSON R, NARUNSKY K, DU BOIS J, VENTER A, et al (PanACEA Consortium). A dose-ranging trial to optimize the dose of rifampin in the treatment of tuberculosis. Am J Respir Crit Care Med. 2015; 191 (9): 1058-65. doi: 10.1164/rccm.201407-1264OC

15.- LONG MW, DE SNIDER, FARER LS US. Public Health Service cooperative trial of three rifampinisoniazid regimens in treatment of tuberculosis. Am. Rev. Respir. Dis.1979; 119: 879-94.

16.- SIRGELF A, FOURIE PB, DONALD PR, PADAYATCHI N, RUSTOMJEE N, LEVIN J, et al. The early bactericidal activities of rifampin and rifapentine in pulmonary tuberculosis. Am. J. Respir. Crit. Care Med. 2005; 172: 128-35.

17.- SVENSSON RJ, NIWARD K, FORSMAN LD, BRUCHFELD J, PAUES J, ELIASSON J, et al. Individualised dosing algorithm and personalised treatment of high-dose rifampicin for tuberculosis. Br J Clin Pharmacol. 2019; 85: 2341-50. DOI:10.1111/bcp.14048

18.- SVENSSON RJ, AARNOUTSE RE, DIACON AH, DAWSON R, GILLESPIE SH, BOEREE MJ, et al. A Population Pharmacokinetic Model Incorporating Saturable Pharmacokinetics and Autoinduction for High Rifampicin Doses. Clin Pharmacol Ther 2018; 103 (4): 674-83. doi: 10.1002/cpt.778.

19.- RAY J, GARDINER I, MARRIOTT D. Managing antituberculosis drug therapy by therapeutic drug monitoring of rifampicin and isoniazid. Intern. Med. J. 2003; 33: 229-34.

20.- PELOQUIN C A. Using therapeutic drug monitoring to dose the antimycobacterial drugs. Clin. Chest Med. 1997; 18: 79-87.

21.- CHAROENRATANAKUL S, DEJSOMRITRUTAI W, CHAIPRASERT. A Diagnostic role of fiberoptic bronchoscopy in suspected smear negative pulmonary tuberculosis. Respiratory Medicine 1995; 89: 621-3.

22.- KUMAR A, GUPTA AK, KHAN MH. Role of flexible fiberoptic bronchoscopy in suspected sputum smear negative pulmonary tuberculosis cases at microscopy centre under RNTCP. Int J Med Sci Public Health. 2014; 3 (1): 31-34. DOI: 10.5455/ijmsph.2013.200920133

23.- SOTO A, SOLARI L, AGAPITO J, GOTUZZO E, ACCINELLI R, VARGAS D, et al. Algorithm for the diagnosis of smear-negative pulmonary tuberculosis in high-incidence resource-constrained settings. Tropical Medicine and International Health. 2013, 18 (10): 122230. doi:10.1111/tmi.12172

24.- PEÑA C, CÉSPED M, WOLFF M, ÁlVAREZ F, GARAY C, MEDINA M, et al. Diagnóstico bacteriológico de tuberculosis pulmonar mediante fibrobroncoscopía en pacientes con VIH. Rev Chil Enferm Respir. 2014; 30: 46-53.

25.- WORLD HEALTH ORGANIZATION. Xpert MTB/ RIF implementation manual: technical and operational 'how-to'; practical considerations. WHO Library Cataloguing-in-Publication Data 2014.

26.- WORLD HEALTH ORGANIZATION. Systematic screening for active tuberculosis: principles and recommendations. WHO Library Cataloguing-in-Publication Data 2013.

27.- KRANZER K, AFNAN-HOLMES H, TOMLIN K, GOLUB JE, SHAPIRO A, SCHAAP A, et al. The benefits to communities and individuals of screening for active tuberculosis disease: a systematic review. Int J Tuberc Lung Dis. 2013; 17 (4): 432-46.

28.- LEWINSOHN DM, LEONARD MK, LOBUE PA, COHN DL, DALEY CL, DESMOND E, et al. Official American Thoracic Society/Infectious Diseases Society of America/Centers for Disease Control and Prevention Clinical Practice Guidelines: Diagnosis of Tuberculosis in Adults and Children. Clinical Infectious Diseases. 2017: 64 (2). DOI: 10.1093/cid/ciw694

29.- DHEDA K, RUHWALD M, THERON G, PETER J, CHEONG W. Point-of-care diagnosis of tuberculosis: Past, present and future. Respirology 2013; 18: 217-32 doi: 10.1111/resp.12022.
Correspondencia a:

Dr. Carlos Peña Mantinetti

Encargado del PROCET,

Servicio de Salud Metropolitano Central.

Hospital Clínico San Borja Arriarán. Santiago, Chile.

Email: carpemanti@hotmail.com 